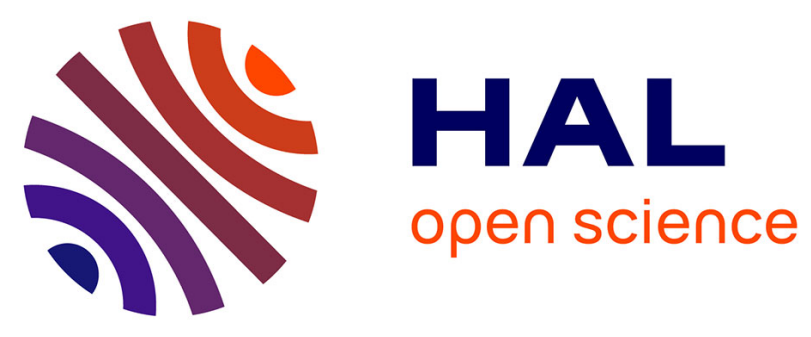

\title{
Valeur pronostique de la TEP/TDM dans le bilan initial du cancer du col utérin: SUV max de la tumeur primitive et stadification ganglionnaire
}

B. Orsal, A. Bourdon, M Benkiran, D de Verbizier, D. Ilonca, Denis

Mariano-Goulart

\section{To cite this version:}

B. Orsal, A. Bourdon, M Benkiran, D de Verbizier, D. Ilonca, et al.. Valeur pronostique de la TEP/TDM dans le bilan initial du cancer du col utérin: SUV max de la tumeur primitive et stadification ganglionnaire. Médecine Nucléaire - Imagerie Fonctionnelle et Métabolique, 2014, 10.1016/j.mednuc.2014.01.001 . hal-02547831

\section{HAL Id: hal-02547831 \\ https://hal.umontpellier.fr/hal-02547831}

Submitted on 20 Apr 2020

HAL is a multi-disciplinary open access archive for the deposit and dissemination of scientific research documents, whether they are published or not. The documents may come from teaching and research institutions in France or abroad, or from public or private research centers.
L'archive ouverte pluridisciplinaire HAL, est destinée au dépôt et à la diffusion de documents scientifiques de niveau recherche, publiés ou non, émanant des établissements d'enseignement et de recherche français ou étrangers, des laboratoires publics ou privés. 


\title{
Valeur pronostique de la TEP/TDM dans le bilan initial du cancer du col utérin : $\mathrm{SUV}_{\max }$ de la tumeur primitive et stadification ganglionnaire
}

\author{
The Prognostic value of initial PET/CT in cervical cancer: $S U V_{\max }$ \\ (standardized uptake value) of the primary tumor and lymph node staging
}

\author{
B. Orsal ${ }^{\text {a }}$, A. Bourdon ${ }^{\text {a }}$, M. Benkiran ${ }^{\text {a }}$, D. De Verbizier ${ }^{\text {b }}$, D. Ilonca ${ }^{\text {b }}$, D. Mariano-Goulart ${ }^{\text {a,* }}$ \\ a Service de médecine nucléaire, CHU Lapeyronie, 371, avenue du Doyen-Gaston-Giraud, 34295 Montpellier cedex 05, France \\ ${ }^{\mathrm{b}}$ Service de médecine nucléaire, CHU Gui-de-Chauliac, 80, avenue Augustin-Fliche, 34000 Montpellier, France
}

\begin{abstract}
Résumé
Objectif. - L'objectif principal était d'évaluer la valeur pronostique de la fixation de ${ }^{18} \mathrm{~F}$-fluorodéoxyglucose de la tumeur primitive mesurée par la Standardized Uptake Value ( $\mathrm{SUV}_{\max }$ ) définie par la tomographie par émission de positons (TEP) dans le cancer du col utérin. L'objectif secondaire était de déterminer les performances diagnostiques de la TEP pour la stadification ganglionnaire pelvienne et lombo-aortique.

Méthode. - Nous avons inclus, rétrospectivement, 49 patientes présentant un cancer cervical utérin de stade FIGO IB1-IVB. La valeur pronostique de la SUV $\mathrm{max}_{\text {ax }}$ a été recherchée par une analyse univariée. La survie a été analysée par la méthode de Kaplan-Meier. L'analyse histologique des ganglions était le gold standard.

Résultats. - Une différence significative des $\mathrm{SUV}_{\max }$ tumorales était observée entre le stade I et II, le stade I et IV et la taille de la tumeur $\leq 4 \mathrm{~cm}$ et $>4 \mathrm{~cm}(p=0,0001)$. Il existait une corrélation entre la $\operatorname{SUV}_{\max }$ et le grand axe de la tumeur $(r=0,597)(p<0,0001)$. L'envahissement ganglionnaire pelvien était prédictif de la survie sans progression $(p=0,0007)$. La valeur prédictive négative (VPN) de la TEP pour les ganglions lombo-aortiques $(n=24)$ était de $100 \%$ dans les stades IB2-IVA. La spécificité et la VPN pour les ganglions pelviens $(n=8)$ étaient de $100 \%$ et $87,5 \%$ (7/8), respectivement, dans les stades précoces. Le ganglion faussement négatif mesurait moins de $2 \mathrm{~mm}$.

Conclusion. - Nos résultats démontrent une corrélation entre la $\mathrm{SUV}_{\max }$ et le grand axe tumoral, qui représente un indicateur de l'agressivité tumorale. La TEP a une bonne VPN pour la stadification ganglionnaire lombo-aortique dans les stades localement avancés. Elle reste cependant insuffisante pour prédire l'atteinte ganglionnaire pelvienne et ne permet pas d'éviter la lymphadénectomie dans les stades précoces.
\end{abstract}

Mots clés : Cancer du col utérin ; TEP/TDM ; $\mathrm{SUV}_{\max }$; Pronostic ; Ganglions ; Stadification

\begin{abstract}
Purpose. - We investigated the prognostic significance of F-18 fluorodeoxyglucose (FDG) uptake measured as maximum Standardized Uptake Value $\left(\mathrm{SUV}_{\max }\right)$ in primary tumor by positron emission tomography/computed tomography (PET/CT) in cervical cancer. The secondary objective was to determine the accuracy of the PET/CT for detecting pelvic lymph node (PLN) and para-aortic lymph node (PALN) metastases.

Methods. - This retrospective study included 49 consecutive patients with stage IB1 to IVB cervical cancer. Univariate analysis was performed to determine the relationships between $\mathrm{SUV}_{\max }$ value and pathological prognostics factors. Survival was estimated by Kaplan-Meier method. The gold standard of LN metastases was histologic.

Results. - A significant difference in $\mathrm{SUV}_{\max }$ was observed between stage I and stage II, stage I and stage IV and tumor size $\leq 4 \mathrm{~cm}$ and $>4 \mathrm{~cm}$ $(P=0.0001)$. There was a significant correlation between the $\mathrm{SUV}_{\max }$ and tumor maximal size $(r=0.597)(P<0.0001)$. PLN metastasis was found to be predictive of progression-free survival $(P=0.0007)$. The negative predictive value $(\mathrm{NPV})$ of the PET/CT for PALN was $100 \%$ for locally advanced
\end{abstract}

\footnotetext{
* Auteur correspondant.

Adressese-mail : blancheorsal@hotmail.fr (B. Orsal),d-mariano_goulart@chu-montpellier.fr (D. Mariano-Goulart).
} 
cervical carcinoma in 24 patients. The specificity and NPV of the PET/CT for PLN in eight early-stage cervical cancer were $100 \%$ and $87.5 \%$ (7/8) respectively. The PET/CT false-negative PLN measured less than $2 \mathrm{~mm}$.

Conclusion. - Our results demonstrate a correlation between $\mathrm{SUV}_{\max }$ and tumor maximal size, which represents an indicator of tumor aggressiveness. PET/CT is effective to predict the absence of PALN in locally advanced cervical carcinoma. PET/CT is not sufficient to predict PLN in early-stage cancer without lymphadenectomy.

Keywords: Cervical cancer; PET/CT; $\mathrm{SUV}_{\max }$; Prognostic; Lymph node

\section{Introduction}

Depuis une quinzaine d'année, l'imagerie occupe une place majeure dans la prise en charge du cancer du col utérin. La prise en charge thérapeutique et le pronostic des patientes dépendent de la classification de la Fédération internationale de gynécologie et obstétrique (FIGO) [1], du type et du grade histologique, de la taille de la tumeur ainsi que du statut ganglionnaire pelvien et lombo-aortique. L'atteinte ganglionnaire lombo-aortique est l'un des facteurs pronostiques majeurs des cancers du col utérin.

Les différentes options thérapeutiques des cancers localisés (stade FIGO 1BI de taille $\leq 4 \mathrm{~cm}$ ) sont la chirurgie, une association radio-chirurgicale ou une radiothérapie externe. Le traitement chirurgical associe une colpo-hystérectomie et une lymphadénectomie pelvienne, étendue en lombo-aortique en cas de curage pelvien positif. Les cancers localement avancés (stade FIGO IB2 à IVA) se définissent par une taille $>4 \mathrm{~cm}$ et/ ou une atteinte extracervicale. Le standard thérapeutique est la radiochimiothérapie concomitante combinant une radiothérapie externe pelvienne associée à une chimiothérapie puis à une curiethérapie endo-cavitaire. En cas d'envahissement des ganglions lombo-aortiques, l'irradiation pelvienne est étendue en lombo-aortique. À ce jour, la lymphadénectomie avec examen histologique des ganglions est la technique de référence pour la stadification ganglionnaire.

La tomographie par émission de positons (TEP) au 18Ffluorodéoxyglucose $\left({ }^{18} \mathrm{FDG}\right)$ représente une alternative intéressante pour évaluer l'envahissement ganglionnaire en évitant la morbidité des curages lombo-aortiques. En effet, plusieurs études ont démontré la supériorité de la TEP au ${ }^{18} \mathrm{~F}$ fluorodéoxyglucose $\left({ }^{18} \mathrm{FDG}\right)$ sur l'imagerie par tomodensitométrie (TDM) ou par résonance magnétique (IRM) pour la stadification ganglionnaire. Ces constatations ont été confirmées dans une méta-analyse publiée en 2010 par Choi et al. [2] qui compare les performances diagnostiques de la TDM, de l'IRM et de la TEP/TDM pour diagnostiquer des ganglions métastatiques dans le cancer du col utérin. La sensibilité et la spécificité étaient, respectivement, de $50 \%$ et $92 \%$ pour la TDM (20 études), de $56 \%$ et $91 \%$ pour l'IRM (31 études) et de $82 \%$ et $95 \%$ pour la TEP/TDM (20 études). Ainsi, la TEP est préconisée depuis 2005 dans les Standards Options et Recommandations (SOR) pour la stadification ganglionnaire [3].

La TEP a une bonne sensibilité de détection de la tumeur cervicale, quel que soit leur type histologique, mais elle reste insuffisante pour le bilan d'extension local [4]. L'IRM demeure l'examen de référence pour préciser la topographie, les dimensions de la tumeur ainsi que son extension locorégionale au sein du pelvis.

Si la place de la TEP est maintenant bien établie dans le cancer du col utérin, la valeur pronostique du paramètre quantitatif de la tumeur cervicale, la Standard Uptake Value maximale $\left(\mathrm{SUV}_{\mathrm{max}}\right)$, doit être confirmée. Dans de nombreuses études, la $\mathrm{SUV}_{\max }$ préthérapeutique de la tumeur apparaissait comme un facteur pronostique de survie [5-7]. En revanche, la corrélation entre le $\mathrm{SUV}_{\max }$ et le stade FIGO, l'envahissement ganglionnaire, la réponse à la chimiothérapie n'était pas retrouvée dans toutes les études et restait controversée [8].

L'objectif principal de notre étude était d'évaluer l'intérêt de la $\mathrm{SUV}_{\max }$ tumorale du col utérin comme facteur pronostique de la maladie.

L'objectif secondaire a consisté à évaluer les performances diagnostiques de la TEP/TDM dans la stadification ganglionnaire pelvienne et lombo-aortique.

\section{Matériel et méthodes}

\subsection{Population}

Nous avons coordonné une étude rétrospective multicentrique menée au CHU de Montpellier, à l'Institut régional du cancer de Montpellier (ICM) et à la Clinique Mutualiste Beau-Soleil de Montpellier entre janvier 2009 et juin $2013 \mathrm{chez}$ des patientes présentant un cancer invasif du col utérin.

Les critères d'inclusion étaient :

- cancer du col utérin confirmé histologiquement sur une biopsie cervicale ;

- stade FIGO $\geq$ IB1 ;

- TEP/TDM dans le bilan initial de stadification ;

- disponibilité des résultats histologiques de l'hystérectomie et des curages ganglionnaires quand ceux-ci ont été réalisés ; - accessibilité aux dossiers de prise en charge.

\subsection{Imagerie et critères d'interprétation de la TEP/TDM au ${ }^{18} \mathrm{FDG}$}

\subsubsection{Conditionnement préalable et installation de la patiente}

La patiente devait être à jeun de plus de $6 \mathrm{~h}$, avec pour cible une glycémie inférieure à $1,80 \mathrm{~g} / \mathrm{L}$. Après l'injection de $5,5 \mathrm{MBq} / \mathrm{kg}$ de ${ }^{18} \mathrm{FDG}$ par voie intraveineuse (Glucotep 
Cyclopharma, Marseille), la patiente était isolée dans un box au calme pendant une heure. Une injection de $20 \mathrm{mg}$ de furosémide était réalisée lors de l'injection du ${ }^{18} \mathrm{FDG}$ en l'absence de contre-indication. L'acquisition TEP/TDM a été réalisée en décubitus dorsal après miction, bras au-dessus de la tête.

\subsubsection{Protocole d'acquisition}

Le recrutement a été multicentrique mais les examens de TEP/TDM ont tous été réalisés au CHU de Montpellier sur la même machine et selon le même protocole d'acquisition. Il s'agit d'un appareil hybride TEP/TDM (Siemens Biograph Emotion duo PET/CT system). La TEP est équipée d'un scintillateur LSO (OrthoSilicate de Lutétium) et d'un scanner double coupe. Le mode d'acquisition a été de type 3D. Le poids des patientes était enregistré pour permettre le calcul de la $\mathrm{SUV}_{\text {max }}$.

Deux acquisitions ont été réalisées en respiration libre :

- la première correspondait à l'acquisition tomodensitométrique (paramètres techniques : $90 \mathrm{mAs}, 110 \mathrm{kV}$, épaisseur de coupe : $5 \mathrm{~mm}$ et incrément de reconstruction : $2,5 \mathrm{~mm}$ ) ;

- la seconde acquisition correspondait aux images TEP (paramètres techniques : 7 à 9 pas de trois minutes chacun avec une matrice de $128 \times 128$ ).

Les sinogrammes 3D ont été reformatés en sinogrammes transverses au moyen d'un algorithme « Fourier-Rebinning ». Les sinogrammes transverses ainsi produits ont ensuite été reconstruits au moyen d'un algorithme OSEM (Ordered Subsets Expectation Maximization) 2D. Les données recueillies et reconstruites ont été visualisées sans et avec correction d'atténuation, fusionnées ou non aux données TDM, dans les trois plans de l'espace et en mode 3D ciné MIP (Maximum Intensity Projection) sur une station « e-soft ».

\subsubsection{Critères d'interprétation}

Une région d'intérêt (ROI) a été tracée manuellement sur la lésion hypermétabolique du col utérin. La $\mathrm{SUV}_{\max }$ a été calculée automatiquement après enregistrement préalable du poids de la patiente.

La fixation ganglionnaire pelvienne et lombo-aortique a été interprétée par rapport au bruit de fond de l'aorte abdominale sous-rénale :

- positive si $\mathrm{SUV}_{\text {max ganglion }} \geq \mathrm{SUV}_{\max \text { aorte }}+0,2$;

- douteuse si $\mathrm{SUV}_{\text {max ganglion }}=\mathrm{SUV}_{\max }$ aorte $\pm 0,2$;

- négative si $\mathrm{SUV}_{\max }$ ganglion $\leq \mathrm{SUV}_{\max \text { aorte }}-0,2$.

\subsection{Traitement des patientes}

Toutes les patientes étaient traitées et suivies de manière standardisée selon les référentiels de la Société française d'oncologie gynécologique [9]. Pour les tumeurs de petit stade (IB1), il n'y avait pas de traitement standard mais différentes options : (a) le traitement chirurgical consistait à réaliser dans un premier temps un curage ganglionnaire pelvien suivi d'une colpo-hystérectomie élargie en cas d'examen extemporané négatif. En présence d'un type histologique agressif ou de ganglions pelviens envahis sur le résultat définitif de l'anatomopathologie, les patientes recevaient un traitement complémentaire. Si l'examen extemporané des ganglions pelviens était positif ou mettait en évidence des emboles vasculaires, les patientes étaient alors traitées par radiochimiothérapie concomitante pelviennne suivie d'une curiethérapie utéro-vaginale et d'une chirurgie de clôture par colpohystérectomie élargie ; (b) le traitement radio-chirurgical consistait à réaliser une curiethérapie préopératoire suivie d'une colpo-hystérectomie élargie 5 à 8 semaines après.

Le traitement standard des cancers localement avancés (IB2IVA) était la radiochimiothérapie concomitante, combinant une radiothérapie externe pelvienne à une chimiothérapie puis à une curiethérapie endo-cavitaire. Si la TEP/TDM ne retrouvait pas de fixation ganglionnaire pathologique à l'étage lomboaortique, un curage de stadification était réalisé. Le curage lombo-aortique remontait de l'axe iliaque primitif aux veines rénales. En cas d'envahissement des ganglions lomboaortiques, l'irradiation pelvienne était étendue en lomboaortique. L'hystérectomie de clôture a été réalisée par voie abdominale ou cœlioscopique dans les 5 à 8 semaines en fonction des résultats de l'imagerie d'évaluation en fin de traitement.

\subsection{Recueil des données}

Le type et le grade histologique tumoral étaient issus des résultats de la biopsie cervicale ou de l'hystérectomie. L'atteinte ganglionnaire était jugée sur le curage ganglionnaire pour estimer les performances diagnostiques de la TEP/TDM.

Le plus grand axe de la tumeur était évalué à partir des données de l'IRM pré-thérapeutique.

Pour les patientes opérées environ 4 à 6 semaines après la fin de l'irradiation, la réponse au traitement a été évaluée par l'analyse histologique de la pièce opératoire. L'existence d'un résidu tumoral était un critère permettant d'apprécier la réponse au traitement initial. La persistance d'un résidu tumoral sur la pièce opératoire était analysée comme une réponse histologique partielle et l'absence de résidu tumoral comme une réponse histologique complète. Quand la chirurgie de clôture n'était pas réalisée, la réponse était basée sur les résultats de l'IRM postthérapeutique. La présence d'un résidu tumoral sur les séquences $\mathrm{T} 1$ et $\mathrm{T} 2$ était considérée comme une réponse partielle et l'absence de résidu comme une réponse complète. La présence d'une atteinte ganglionnaire comme facteur prédictif de survie sans progression était jugée sur les résultats histologiques et les résultats de la TEP/TDM.

\subsection{Suivi des patientes}

Le suivi était fait par un médecin spécialiste en gynécologie ou en oncologie-radiothérapie. La durée de suivi correspondait au délai depuis le diagnostic de la maladie tumorale à la dernière consultation réalisée par un de ces spécialistes. La 
patiente était considérée en progression en cas d'augmentation de taille significative des lésions déjà connues ou en cas d'apparition de lésions secondaires ganglionnaires ou à distance par rapport au bilan d'extension initial sur un examen d'imagerie IRM ou TDM (critères RECIST) ou TEP/TDM.

\subsection{Analyse statistique}

Les capacités intrinsèques et extrinsèques de la TEP/TDM étaient calculées pour l'atteinte ganglionnaire pelvienne et lombo-aortique par ganglion et par patiente. Du fait de la distribution non gaussienne du paramètre, la liaison statistique entre la $\mathrm{SUV}_{\max }$ et les facteurs pronostiques de la maladie était évaluée par un test non paramétrique de Mann-Whitney à partir du logiciel Prism 6 de GraphPad. La corrélation entre la taille de la tumeur et la $S U V_{\max }$ était analysée à partir du coefficient de corrélation de Spearman. Les variables qualitatives étaient exprimées en pourcentages et une comparaison entre les groupes était effectuée à partir du test du $\mathrm{Chi}^{2}$. La méthode de Kaplan-Meier était utilisée pour estimer la survie sans progression. Le seuil de significativité était prédéfini à $p<0,05$.

\section{Résultats}

\subsection{Analyse descriptive des patientes}

Quarante-neuf patientes de stade FIGO IB1 à IVB ont été incluses et présentaient un âge moyen de 53 ans [31,5-80,8]. Le délai moyen entre l'injection et l'acquisition a été de 69 minutes [53-115 min]. La glycémie moyenne à l'injection était de 5,12 mmol/L $(0,93 \mathrm{~g} / \mathrm{L})$.

Tableau 1

Caractéristiques des patientes $(n=49)$.

Patient characteristics $(n=49)$.

\begin{tabular}{llr}
\hline Caractéristiques & $n=49$ & $\%$ \\
\hline Âge (moyen et extrêmes) & 53 & \\
Stade FIGO (n) & $(31,5-0,8)$ & \\
IB1 & & 16,3 \\
IB2 & 8 & 6,1 \\
IIA & 3 & 8,2 \\
IIB & 4 & 44,9 \\
IIIA & 22 & 0,0 \\
IIIB & 0 & 2,0 \\
IVA & 1 & 4,1 \\
IVB & 2 & 18,4 \\
Histologie (n) & 9 & \\
Carcinome épidermoïde & & 65,3 \\
Adénocarcinome & 32 & 32,7 \\
(dont cellules claires) & 16 & \\
Adénosquameux & $(3)$ & 2,0 \\
Grade (n) & 1 & 12,2 \\
1 & & 40,8 \\
2 & 6 & 32,7 \\
3 & 20 & 14,3 \\
Non mentionné & 16 & \\
\hline
\end{tabular}

La classification de FIGO et l'histologie des lésions cervicales initiales sont résumées dans le Tableau 1.

Les résultats histologiques de la tumeur primitive étaient disponibles pour l'ensemble des patientes. Le grade histologique n'a pas pu être analysable chez 7 patientes en raison d'un matériel histologique insuffisant $(n=42)$. Pour une patiente, la TEP/TDM n'a pas mis en évidence la tumeur cervicale. La sensibilité de la TEP/TDM pour détecter la tumeur primitive était de $98 \%(48 / 49)$.

\section{2. Évaluation de la $S U V_{\max }$ tumorale comme facteur pronostique}

La $\mathrm{SUV}_{\max }$ moyenne de la tumeur primitive était de $11,27 \pm 6,45$ pour tous les stades confondus.

Les $\mathrm{SUV}_{\max }$ de la tumeur en fonction des facteurs pronostiques sont présentées pour la totalité de la série dans le Tableau 2. La valeur moyenne du stade III n'était pas analysable en raison d'un effectif insuffisant $(n=1)$.

Tableau 2

$\mathrm{SUV}_{\text {max }}$ de la tumeur cervicale en fonction des facteurs pronostiques. $S U V_{\text {max }}$ of primary cervical tumor stratified by prognostic factors.

\begin{tabular}{|c|c|c|c|}
\hline Facteurs pronostiques & $n$ & $\begin{array}{l}\mathrm{SUV}_{\max } \\
\pm \text { écart-type }\end{array}$ & $p$ \\
\hline \multicolumn{4}{|l|}{$\hat{A} g e$ (ans) } \\
\hline$<50$ & 24 & $10,54 \pm 7,02$ & \multirow[t]{2}{*}{$p=0,267$} \\
\hline$\geq 50$ & 25 & $11,98 \pm 6,08$ & \\
\hline \multicolumn{4}{|l|}{ Taille de la tumeur $(\mathrm{cm})$} \\
\hline$\leq 4$ & 20 & $7,9 \pm 3,82$ & \multirow[t]{3}{*}{$p=0,0001$} \\
\hline$>4$ & 22 & $14,95 \pm 6,82$ & \\
\hline Non disponible & 7 & & \\
\hline \multicolumn{4}{|l|}{ Stade FIGO } \\
\hline I & 11 & $7 \pm 4,42$ & \multirow{4}{*}{$\begin{array}{l}p=0,0108 \\
\text { entre les } \\
\text { stades I et II } \\
p=0,0192 \text { entre } \\
\text { les stades I et IV }\end{array}$} \\
\hline II & 26 & $12,63 \pm 7,53$ & \\
\hline III & 1 & 13,83 & \\
\hline IV & 11 & $12,11 \pm 4,14$ & \\
\hline \multicolumn{4}{|l|}{ Type histologique } \\
\hline Carcinome épidermoïde & 32 & $10,85 \pm 6,42$ & \multirow[t]{2}{*}{$p=0,986$} \\
\hline Adénocarcinome & 16 & $12,36 \pm 6,97$ & \\
\hline \multicolumn{4}{|l|}{ Grade histologique } \\
\hline $\begin{array}{l}\text { Bien/moyennement } \\
\text { différencié }\end{array}$ & 26 & $12 \pm 6,02$ & \multirow[t]{3}{*}{$p=0,266$} \\
\hline Mal différencié & 16 & $11,2 \pm 8,07$ & \\
\hline Non disponible & 7 & & \\
\hline \multicolumn{4}{|l|}{ Ganglions pelviens } \\
\hline N0 & 29 & $10,74 \pm 7,0$ & \multirow[t]{2}{*}{$p=0,3637$} \\
\hline $\mathrm{N} 1$ & 20 & $12,05 \pm 5,86$ & \\
\hline \multicolumn{4}{|l|}{ Résidu tumoral } \\
\hline $\mathrm{R}-$ & 16 & $9,16 \pm 4,6$ & \multirow[t]{3}{*}{$p=0,093$} \\
\hline $\mathrm{R}+$ & 25 & $13,06 \pm 6,62$ & \\
\hline Non disponible & 8 & & \\
\hline \multicolumn{4}{|l|}{ Progression } \\
\hline Absence & 28 & $10,67 \pm 5,33$ & \multirow[t]{3}{*}{$p=0,623$} \\
\hline Présence & 17 & $11,52 \pm 6,93$ & \\
\hline Non disponible & 4 & & \\
\hline
\end{tabular}


L'envahissement ganglionnaire pelvien était évalué sur les données de la TEP/TDM chez 41 patientes non opérées d'emblée et à partir du curage ganglionnaire chez 8 patientes. Les ganglions pelviens étaient positifs chez 20 patientes et négatifs chez 29 patientes. Toutes les patientes avec un envahissement ganglionnaire lombo-aortique $(n=9)$ présentaient une atteinte ganglionnaire pelvienne.

Pour 8 des 49 patientes, la présence d'un résidu tumoral après radiochimiothérapie n'était pas analysable : 6 patientes présentant des tumeurs de stades localisés ont été traitées par chirurgie d'emblée et 2 patientes, en cours de traitement, n'ont pas eu d'évaluation post-thérapeutique ou de chirurgie de clôture. Chez les 41 patientes évaluables, un résidu tumoral était présent $(\mathrm{R}+)$ chez 25 patientes et absent $(\mathrm{R}-)$ chez 16 patientes. L'évaluation a été basée sur les données de l'imagerie chez 12 patientes et sur les résultats anatomopathologiques de l'hystérectomie de clôture chez 29 patientes.

\subsection{Corrélation entre la $S U V_{\max }$ tumorale et la taille de la tumeur}

Nous disposions de la taille de la tumeur déterminée sur l'IRM initiale à partir des séquences morphologiques standard chez 42 patientes. La lésion tumorale de plus petite taille mesurait $10 \mathrm{~mm}$ de grand axe et la plus volumineuse $90 \mathrm{~mm}$ de grand axe.

Le facteur $r$ de corrélation sur 42 paires a été estimé à 0,5973 (IC95 \% : 0,3503-0,7667) $(p<0,0001)$.

Ce résultat est illustré par la courbe de corrélation sur la Fig. 1.

\subsection{Progression tumorale}

Parmi les 49 patientes, 3 ont été perdues de vue lors du suivi et une patiente était en cours de traitement. Le suivi moyen des 45 patientes était de 16,1 mois [3-59 mois]. Une progression a été observée chez 17 patientes au bout d'une durée moyenne de 19 mois [3-53 mois]. Vingt-huit patientes n'ont pas présenté de progression pour un suivi moyen de 12 mois [4,6-22,5 mois].

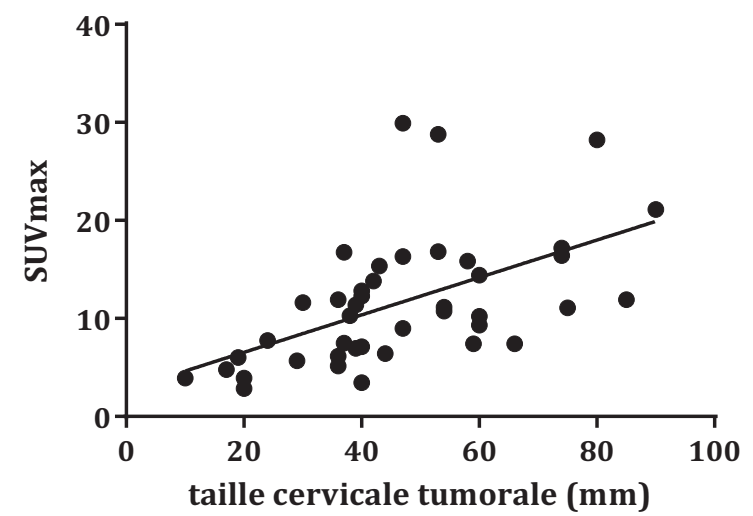

Fig. 1. Relation entre la $\mathrm{SUV}_{\max }$ et le grand axe de la tumeur cervicale (mm). Relationship between $S U V_{\max }$ and the long axis of the cervical cancer ( $\mathrm{mm}$ ).
Il n'existe pas de lien statistiquement significatif entre la progression carcinologique et l'âge des patientes, la taille de la tumeur, le stade FIGO, un $\mathrm{SUV}_{\text {max }}<$ ou $\geq 11,27\left(\mathrm{SUV}_{\max }\right.$ moyenne de la tumeur primitive), le type ainsi que le grade histologique et la réponse à la radiochimiothérapie initiale (Tableau 3). La progression était statistiquement différente entre les patientes présentant ou non une atteinte ganglionnaire $(p=0,0008)$.

La survie sans progression était différente pour les patientes avec ou sans atteinte ganglionnaire $(p=0,0007)$. Chez les patientes évolutives sur le plan carcinologique, 13 avaient initialement une atteinte ganglionnaire et 4 n'avaient pas d'envahissement ganglionnaire. Les 28 patientes sans progression tumorale avaient pour 5 d'entre elles une atteinte ganglionnaire pelvienne. La survie sans progression à 3 ans était de $77,6 \%$ pour les patientes $\mathrm{N}$ - et de $10,6 \%$ pour les patientes $\mathrm{N}+$ (Fig. 2).

Tableau 3

Facteurs pronostiques de progression.

Prognostics factors for progression.

\begin{tabular}{|c|c|c|}
\hline Facteurs pronostiques & $n$ & $p$ \\
\hline \multicolumn{3}{|l|}{$\hat{A} g e($ ans) } \\
\hline$<50$ & 21 & \multirow[t]{3}{*}{$p=0,06$} \\
\hline$\geq 50$ & 24 & \\
\hline Non disponible & 4 & \\
\hline \multicolumn{3}{|l|}{ Taille de la tumeur (cm) } \\
\hline$\leq 4$ & 18 & \multirow{3}{*}{$p=0,2$} \\
\hline$>4$ & 21 & \\
\hline Non disponible & 10 & \\
\hline \multicolumn{3}{|l|}{$F I G O$} \\
\hline Localisé (IB1) & 6 & \multirow[t]{4}{*}{$p=0,1$} \\
\hline Localement avancé (IB1-IVA) & 30 & \\
\hline Métastatique (IVB) & 9 & \\
\hline Non disponible & 4 & \\
\hline \multicolumn{3}{|l|}{$S U V_{\max }$} \\
\hline SUV < 11,27 & 27 & \multirow[t]{3}{*}{$p=0,9$} \\
\hline $\mathrm{SUV} \geq 11,27$ & 18 & \\
\hline Non disponible & 4 & \\
\hline \multicolumn{3}{|l|}{ Type histologique } \\
\hline Carcinomes épidermoïdes & 28 & \multirow[t]{3}{*}{$p=0,9$} \\
\hline Adénocarcinomes & 16 & \\
\hline Non disponible & 4 & \\
\hline \multicolumn{3}{|l|}{ Grade histologique } \\
\hline Bien/moyennement différencié & 22 & \multirow{3}{*}{$p=0,9$} \\
\hline Mal différencié & 16 & \\
\hline Non disponible & 11 & \\
\hline \multicolumn{3}{|l|}{ Ganglions pelviens } \\
\hline N0 & 25 & \multirow[t]{3}{*}{$p=0,0008$} \\
\hline N1 & 20 & \\
\hline Non disponible & 4 & \\
\hline \multicolumn{3}{|l|}{ Résidu tumoral } \\
\hline Absence & 15 & \multirow[t]{3}{*}{$p=0,15$} \\
\hline Présence & 24 & \\
\hline Non disponible & 10 & \\
\hline
\end{tabular}

Étude de la TEP/TDM pour la détection des ganglions lombo-aortiques dans les cancers du col utérin localement avancés. 


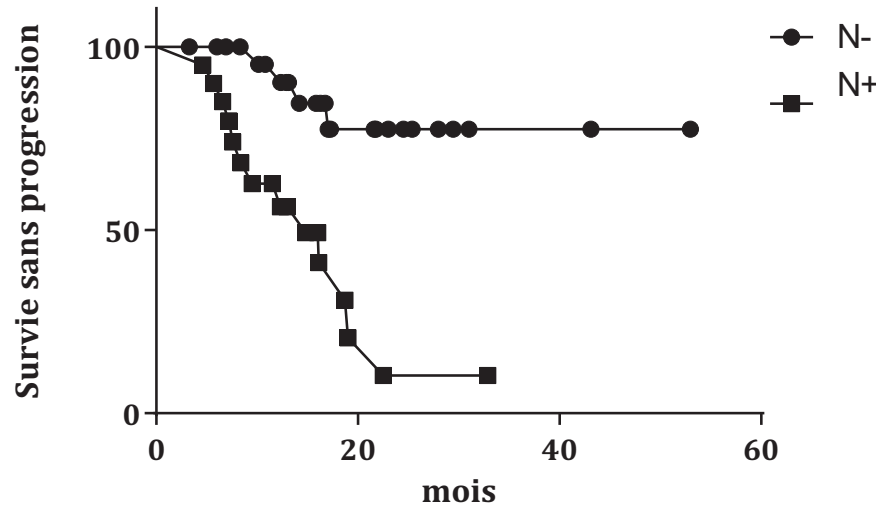

Fig. 2. Survie sans progression en fonction de la présence $(\mathrm{N}+)$ ou l'absence $(\mathrm{N}-)$ de ganglions pelviens $(n=45)$.

Kaplan-Meier progression-free survival with $(N+)$ or without $(N-)$ pelvic lymph node metastasis.

\section{5. Évaluation ganglionnaire en TEP/TDM}

\subsubsection{Stadification ganglionnaire pelvienne}

Les 8 patientes présentant un stade précoce (IB1) ont été prises en charge de la manière suivante (Fig. 3) : 8 patientes ont eu une chirurgie pelvienne première avec hystérectomie et curage ganglionnaire pelvien (stade IB1, $n=6$; stade IB2, $n=2$ ). Pour ces patientes, la TEP/TDM était négative dans les régions pelviennes. L'histologie a retrouvé pour une patiente une métastase ganglionnaire iliaque droite isolée mesurant moins de $2 \mathrm{~mm}$ de grand axe. Il s'agissait d'une tumeur primitive de type adénocarcinome bien différencié de stade IB1. La prévalence de l'atteinte ganglionnaire pelvienne dans les stades localisés a été évaluée à 12,5\% (1/8). La spécificité et la valeur prédictive négative de la TEP sont estimées, respectivement, par patiente à $100 \%(7 / 7)$ et à $87,5 \%(7 / 8)$ et par ganglions à $100 \%(124 / 124)$ et à 99,2 \% (124/125). Aucun des ganglions pelviens n'était positif en TEP ainsi la sensibilité et la valeur prédictive positive n'ont pas pu être évaluées.

\subsubsection{Stadification ganglionnaire lombo-aortique}

Pour les stades IB1 $(n=8)$, la TEP/TDM ne montrait pas de fixation suspecte en lombo-aortique. Aucune des patientes IB 1 n'avait de ganglions pelviens positifs lors de l'extemporané $(n=6)$, ainsi aucun curage ganglionnaire lombo-aortique n'a été réalisé.

Concernant les stades métastatiques IVB $(n=9)$, toutes les patientes avaient des ganglions positifs en lombo-aortiques, mais aucun curage n'a été réalisé conformément aux recommandations.

Pour les patientes présentant un stade FIGO IB2 à IVA, localement avancé $(n=32)$, un examen TEP était positif et une TEP était douteuse dans la région lombo-aortique. Pour les 30 patientes dont la TEP était négative, 24 patientes ont eu un curage lombo-aortique de stadification dont l'histologie ganglionnaire était négative (Fig. 4). Aucun des 329 ganglions analysés n'était histologiquement envahi. Le curage ganglionnaire chirurgical n'a pas été réalisé en cas de comorbidité pour 4 patientes ou en raison d'une chirurgie $1^{\text {re }}$ pelvienne de Wertheim pour 2 patientes traitées en 2010.

Pour la patiente dont la fixation était douteuse en TEP/TDM, l'hypermétabolisme ganglionnaire des 3 ganglions était compris entre la $\mathrm{SUV}_{\max }$ aorte sous-rénale $\pm 0,2$ (Fig. 5). La lymphadénectomie lombo-aortique a mis en évidence 3 métastases ganglionnaires sans rupture capsulaire sur 9 ganglions prélevés. Nous ne connaissons pas la taille des métastases au sein des ganglions envahis. L'analyse par patiente et par ganglions retrouve une valeur prédictive négative de $100 \%$ de la TEP si l'on considère seulement les TEP négatives. Si l'on

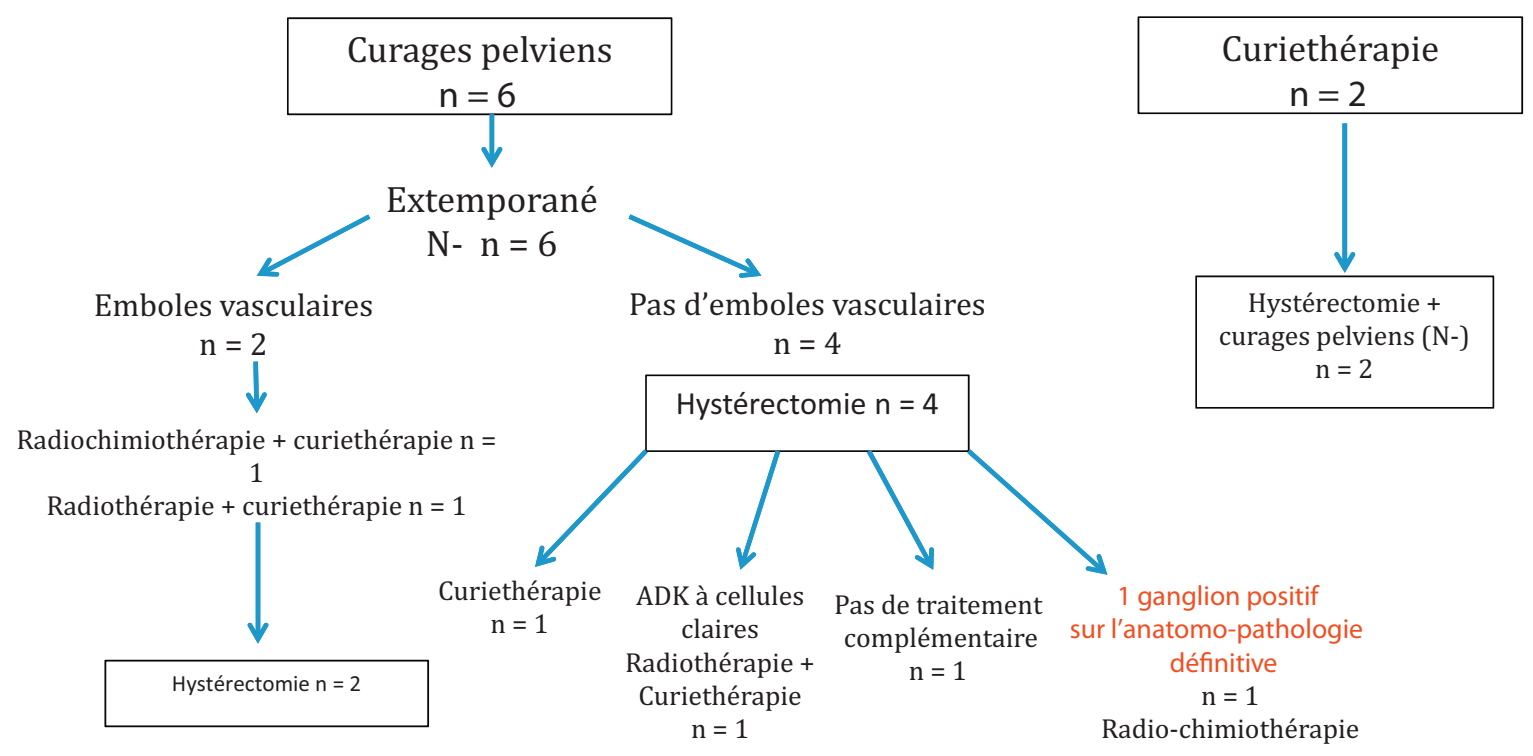

Fig. 3. Prise en charge des patientes atteintes d'un cancer du col utérin de stade FIGO IB1 $(n=8)$.

Management of patients with FIGO stage IB1 cervical cancer $(n=8)$. 


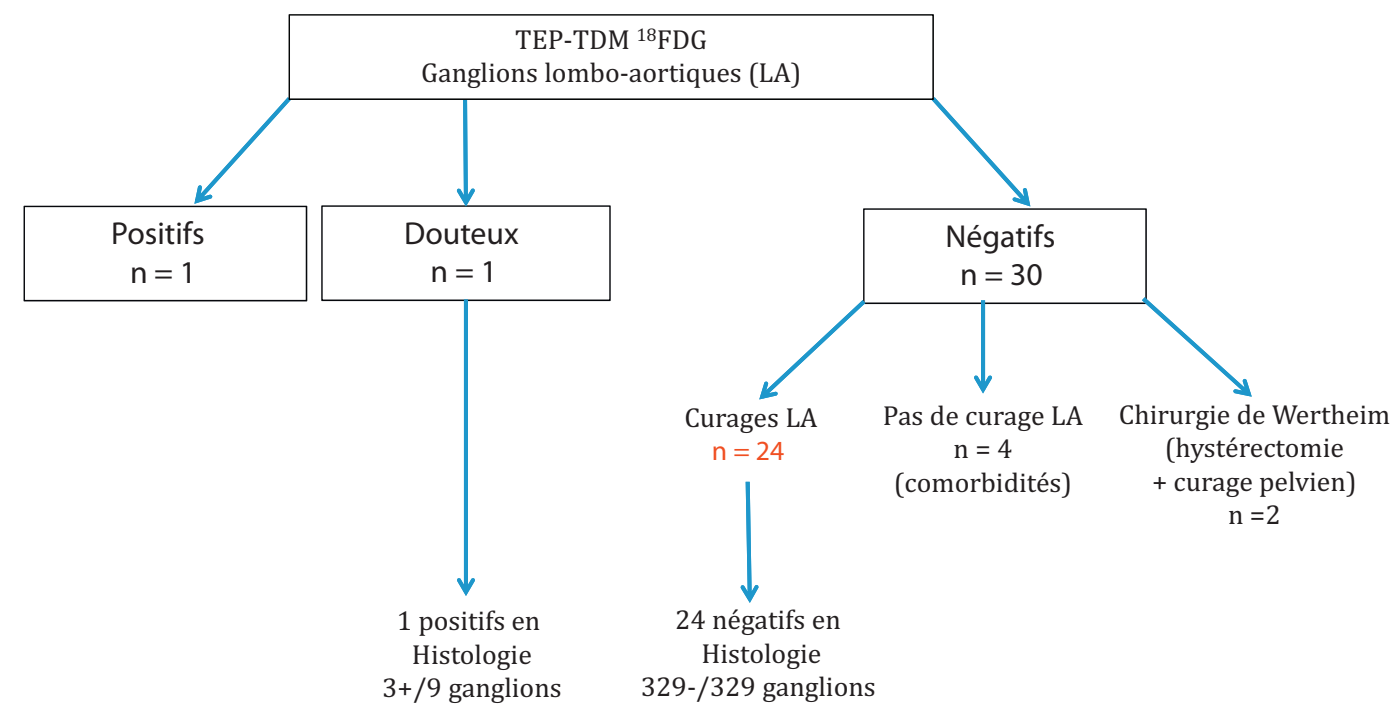

Fig. 4. Prise en charge des patientes atteintes d'un cancer du col utérin de stade FIGO IB2-IVA en fonction des résultats de la TEP/TDM dans la région lomboaortique $(n=32)$.

Management of patients with FIGO stages IB2-IVA according to PET/CT results in the para-aortic area.

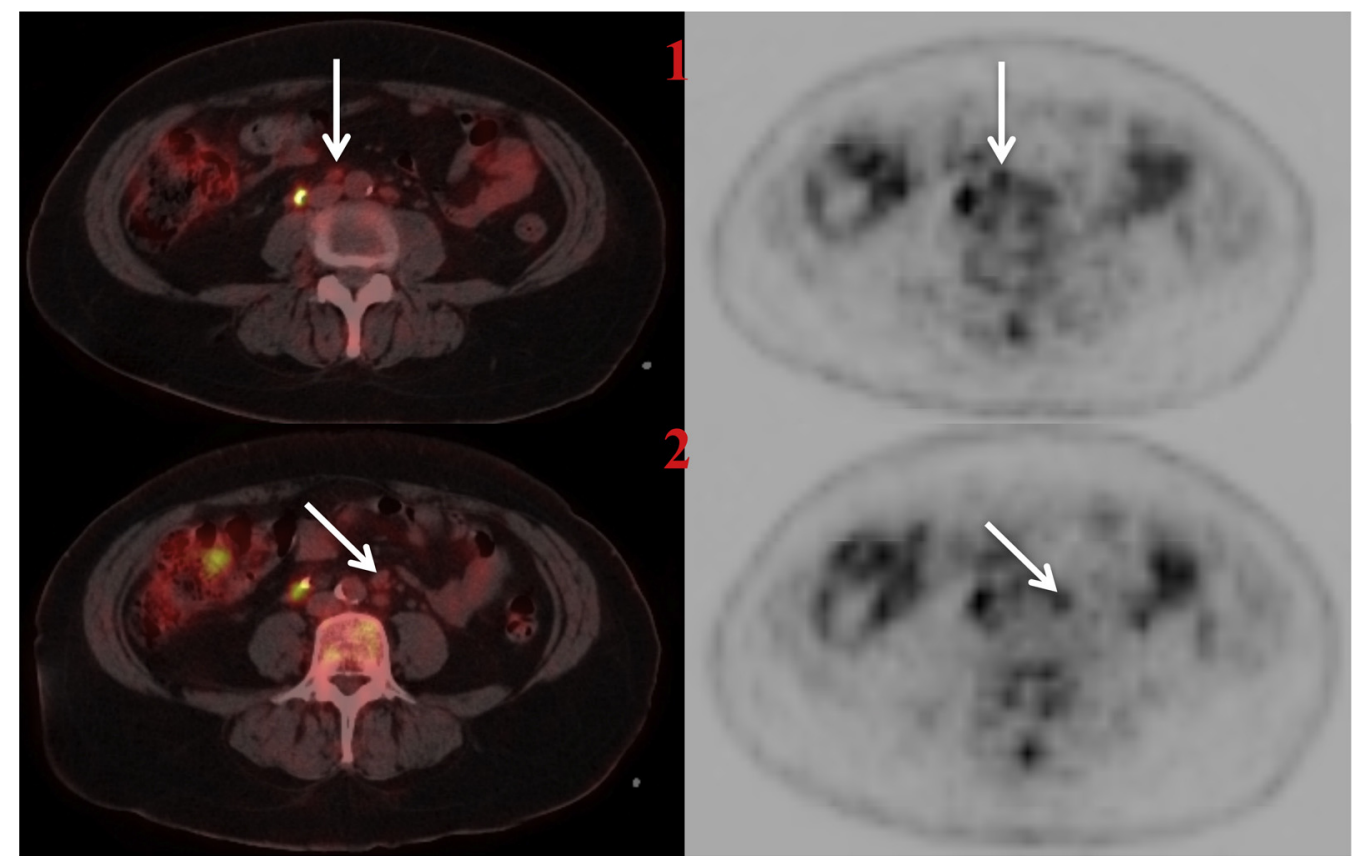

Fig. 5. Ganglions métastatiques douteux dans la région lombo-aortique sur les acquisitions TEP/TDM. $1:$ ganglion inter-aortico-cave $: \mathrm{SUV}$ max $=2,25$ et 7 mm de petit axe ; 2 : ganglions latéro-aortiques : $\mathrm{SUV}_{\max }=2$ et $7 \mathrm{~mm}$ de petit axe et $\mathrm{SUV}_{\max }=2,09$ et 7 mm de petit axe $; \mathrm{SUV}_{\max }$ aorte sous-rénale $=2,13$.

Uncertain lymph node metastasis in the para-aortic area on PET/CT. 1: inter-aorto-caval lymph node: SUV $V_{\text {max }}=2.25$ and 7 mm in short-axis; $2:$ left para-aortic lymph node: $S U V_{\max }=2$ and $7 \mathrm{~mm}$ in short-axis and $S U V_{\max }=2.09$ and $7 \mathrm{~mm}$ in short-axis; $S U V_{\max }$ aorta below the kidneys $=2.13$.

prend en compte les examens négatifs et l'examen douteux, la valeur prédictive négative par patiente et par ganglion était alors, respectivement, de $96 \%(24 / 25)$ et de $99,1 \%(335 /$ 338). Le curage ganglionnaire lombo-aortique a été réalisé en cas d'examen TEP négatif et seule la VPN a pu être calculée.

\section{Discussion}

Le taux de détection de la TEP/TDM a été de $98 \%$ dans notre étude (48/49 patientes), conformément aux données de la littérature où la sensibilité varie de 91 à $97 \%$ [6,10,11]. Le cas non détecté par la TEP/TDM correspondait à un carcinome 
épidermoïde moyennement différencié de stade IB2 $(\geq 4 \mathrm{~cm})$ sans résidu tumoral sur la pièce d'hystérectomie après radiochimiothérapie concomitante et sans récidive après un suivi de 6,9 mois. L'analyse de la région cervicale n'était pas gênée par une accumulation vésicale du radiotraceur. Les différentes hypothèses pour expliquer la non-visualisation de la tumeur sont l'absence d'expression du transporteur du glucose (GLUT-1) par la tumeur qui ne permet pas l'accumulation du ${ }^{18}$ FDG dans la cellule [12] ou encore un défaut de synthèse de l'hexokinase, enzyme essentielle à la phosphorylation du ${ }^{18}$ FDG [13].

On retrouve dans notre étude une différence significative de la $\mathrm{SUV}_{\max }$ entre les patientes de stade FIGO I et II ( $\left.p=0,0108\right)$ et FIGO I et IV ( $p=0,0192)$.

La moyenne des $\mathrm{SUV}_{\max }$ des tumeurs cervicales tend à être différente entre les patientes en réponse histologique complète $(8,32)$ et celles en situation de réponse histologique partielle $(13,55)(p=0,093)$ après radiochimiothérapie concomitante même si cette différence n'est pas considérée comme significative pour un $p<0,05$ et ce malgré l'hétérogénéité des traitements reçus. En effet, sur les 41 patientes analysées, 24 patientes étaient traitées par radiochimiothérapie complétée d'une curiethérapie, 8 patientes par radiothérapie associée à une curiethérapie, 4 patientes par radiothérapie seule, 3 patientes par radiochimiothérapie et 2 patientes par curiethérapie. Ces données seront à valider sur des effectifs plus conséquents.

Ces résultats ont été évoqués dans les travaux de Kidd et al. [7] en 2007 qui ont évalué rétrospectivement la valeur de la $\mathrm{SUV}_{\text {max }}$ pré-thérapeutique et son association à la réponse au traitement et au pronostic chez 287 patientes atteintes de cancer du col de stade IA2 à IVB. La SUV max était un marqueur prédictif du statut ganglionnaire tout comme l'ont suggéré Merlin et al. [14], de la persistance de maladie résiduelle après traitement, d'une récidive pelvienne et de la survie globale. Le taux de survie globale à cinq ans était de $95 \%$ lorsque la $\mathrm{SUV}_{\max }$ était inférieure à 5,2 ; de $70 \%$ pour une $\mathrm{SUV}_{\max }$ comprise entre 5,2 et 13,3 et de seulement $44 \%$ lorsque la $\mathrm{SUV}_{\text {max }}$ était supérieure à $13,3(p<0,0001)$. De plus, Leseur et al. [6] se sont intéressés à la valeur pronostique de la TEP pour la réponse au traitement dans une étude rétrospective incluant 90 patientes traitées pour un cancer du col utérin stade IA2 à IVA FIGO. En analyse multivariée, le diamètre maximal estimé sur la TEP et la présence d'un résidu post-thérapeutique avaient un impact sur la survie sans maladie (respectivement, $p=0,02$ et $p=0,009$ ) et sur la survie globale (respectivement, $p=0,04$ et $p=0,02)$. La moyenne des $\mathrm{SUV}_{\max }$ était significativement différente entre les patientes en réponse histologique complète $(14,1)$ et celles en situation de réponse histologique partielle $(18,9) \quad(p=0,01)$. La fréquence d'un résidu sur la pièce opératoire était augmentée pour les tumeurs ayant une $\mathrm{SUV}_{\max }$ supérieure à 10,9 $(p=0,017)$.

D'autre part, Pan et al. [15] ont montré que la $S_{\text {max }}$ de la tumeur cervicale était différente entre les lésions de taille $\leq 4 \mathrm{~cm}$ et celles $>4 \mathrm{~cm}$.

Contrairement aux différents travaux publiés, la $\mathrm{SUV}_{\max }$ initiale de la tumeur ne représente pas un facteur pronostique de survie sans progression [6-8,16], d'atteinte ganglionnaire [7], de type et de grade histologique [17] dans notre étude. Une des explications possible est que la fixation de ${ }^{18} \mathrm{FDG}$ est plutôt corrélée à l'agressivité de la tumeur cervicale, mais pas à l'extension de la maladie à distance. Toutefois, un des facteurs limitant important de notre étude est le faible effectif de patientes incluses.

La corrélation n'explique pas la causalité, mais le coefficient $r=0,5973$ calculé dans notre étude suggère une relation entre la $\mathrm{SUV}_{\text {max }}$ et la taille de la tumeur. Nos données de $\mathrm{SUV}_{\text {max }}$ au niveau de la lésion cervicale étaient peu soumises à un effet de volume partiel dans la mesure où les deux plus petites lésions mesuraient $10 \mathrm{~mm}$ et $17 \mathrm{~mm}$ de grand axe. Cette corrélation peut s'expliquer par l'association entre la $\mathrm{SUV}_{\max }$ et la prolifération cellulaire et par conséquent la taille de la tumeur. Nguyen et al. [18] suggéraient qu'il existait une corrélation entre la $\mathrm{SUV}_{\max }$ et le Ki67, la SUV $\mathrm{Sax}_{\text {max }}$ et la quantité de GLUT1 ainsi que l'expression de GLUT-1 et le Ki67. Le Ki67 est un marqueur de prolifération présent au niveau du noyau des cellules en multiplication [19].

La croissance d'une tumeur peu différenciée sera plus rapide que celle d'une tumeur différenciée par échappement aux mécanismes normaux de régulation de la croissance cellulaire. Il a été évoqué que l'avidité en ${ }^{18} \mathrm{FDG}$ de la tumeur était corrélée à sa différenciation cellulaire [17] et donc, par conséquent, à sa taille. Toutefois, l'association entre la prolifération cellulaire et la captation de ${ }^{18}$ FDG est controversée. Dans les cancers cérébraux, cervicaux et les lymphomes, la prolifération cellulaire semble associée à une forte accumulation de ${ }^{18} \mathrm{FDG}$ alors qu'elle ne l'est pas dans le cancer du pancréas [20].

En contradiction avec nos résultats, pour Kidd et al. [17], la $\operatorname{SUV}_{\text {max }}$ de la tumeur n'est pas corrélée $\left(r^{2}=0,013\right)$ au volume tumoral chez 240 patientes présentant un cancer du col utérin de stade FIGO IB1 à IVB.

En accord avec les données de la littérature [21,22], l'atteinte ganglionnaire pelvienne dans notre étude était corrélée à la survie sans progression $(p=0,007)$.

L'atteinte ganglionnaire est un facteur pronostique majeur, bien que non pris en compte dans la classification de la FIGO. L'IRM peut mettre en évidence le long des axes vasculaires les ganglions qui sont considérés comme pathologiques lorsque leur petit axe est supérieur à $8-10 \mathrm{~mm}[23,24]$. Le seuil à appliquer dans la caractérisation des ganglions est un sujet souvent débattu. Morisawa et al. [25] ont montré, dans les cancers uro-génitaux, que le seuil de $8 \mathrm{~mm}$ de petit axe pour un ganglion pouvait être retenu comme pathologique. Les techniques radiologiques, purement morphologiques, trouvent leur limite dans la caractérisation des lésions ganglionnaires. Il existe un risque de faux négatif pour les ganglions métastatiques de petite taille $(<8$ ou $10 \mathrm{~mm})$ et un risque de faux positif pour les ganglions inflammatoires. En prenant une taille limite de $10 \mathrm{~mm}$ de petit axe pour classer les ganglions comme pathologiques, l'IRM est moins performante que la TEP/TDM [2].

Dans notre étude, une seule des 8 patientes de stades IB1 $(n=6)$ et 1B2 $(n=2)$ ayant eu un curage pelvien présentait une atteinte ganglionnaire. Cette patiente avait une atteinte qui 
n'avait pas été visualisée en TEP/TDM et correspondait à une micrométastase de moins de $2 \mathrm{~mm}$. L'évaluation ganglionnaire par TEP seule risque donc de méconnaître un envahissement ganglionnaire micrométastatique qui relèverait d'une irradiation.

Le taux de micrométastases pelviennes dans les stades localisés diagnostiqués par immunohistochimie varie entre $4 \%$ et $22 \%$ selon les études [26-28]. Sironi et al. [29] ont été les premiers à s'intéresser à la stadification ganglionnaire pelvienne en TEP/TDM chez 47 patientes atteintes de cancer du col de l'utérus de stade FIGO IA1 $(n=4)$, IB1 $(n=35)$ et IB2 $(n=8)$, dans une étude prospective avec des données de curage ganglionnaire. Les auteurs ont trouvé une sensibilité de la TEP/TDM de $72 \%$, une spécificité de 99,7\%, une valeur prédictive positive de $81 \%$, une valeur prédictive négative de $99,5 \%$ et un taux de détection de 99,3\% par ganglion. Les cinq faux négatifs correspondaient à des métastases ganglionnaires ayant un petit axe inférieur à $5 \mathrm{~mm}$. Les valeurs correspondantes pour la détection des ganglions de plus de $5 \mathrm{~mm}$ de petit axe étaient alors de $100 \%, 99,6 \%, 81 \%, 100 \%$ et $99,6 \%$.

Dans l'étude prospective de Chou [30], qui analysait 60 patientes de stade IA2 à IIA de FIGO avec une prédominance pour le stade 1B1 (90\% des patientes), la sensibilité de la TEP/ TDM pour la détection des métastases ganglionnaires pelviennes était seulement de $10 \%$ (90\% de faux négatifs). Les implants carcinologiques au sein des ganglions non détectés par la TEP/ TDM mesuraient entre $0,5 \times 0,5 \mathrm{~mm}$ et $7 \times 6 \mathrm{~mm}$, ce qui explique le faible taux de détection.

Ainsi dans les petits stades, la lymphadénectomie pelvienne reste indispensable quels que soient les résultats de la TEP/ TDM et de l'imagerie par IRM ou TDM du fait du risque de micrométastases. La technique du ganglion sentinelle pourrait être une alternative au curage systématique. La pratique de coupes sériées et de techniques d'immunohistochimie permettrait d'améliorer la sensibilité de détection de l'analyse histologique en mettant en évidence des micrométastases non détectées par l'examen standard en coloration hématoxyline-éosine safran [31].

Le faible effectif des cancers de stades 1B1 de FIGO et l'absence d'atteinte ganglionnaire en TEP/TDM dans notre étude ne nous ont pas permis de calculer la sensibilité et la valeur prédictive positive de la TEP.

La connaissance des sites de fixation physiologique du ${ }^{18} \mathrm{FDG}$ et les variantes physiologiques de sa distribution sont importantes pour éviter les erreurs d'interprétation de l'examen. Un interrogatoire rigoureux des patientes doit faire préciser la période du cycle ovulatoire. La fixation ovarienne physiologique est fréquemment retrouvée en période ovulatoire et en début de phase lutéale (entre le $10^{\mathrm{e}}$ et le $24^{\mathrm{e}}$ jour) chez les femmes en âge de procréer [32] (Fig. 6). Cette fixation peut être évitée en programmant l'examen en première partie de cycle. Elle n'est pas observée chez les femmes ménopausées. Une fixation de ${ }^{18}$ FDG en regard des ovaires peut également témoigner d'un kyste folliculaire ou d'une hémorragie dans le corps jaune. L'acquisition TEP couplée à la TDM de repérage doit permettre toutefois de réduire ce type d'erreur.

Dans notre étude, la TEP/TDM a eu une très bonne valeur prédictive négative pour les ganglions lombo-aortiques, estimée à $100 \%$ par patiente et par ganglion pour les 24 patientes de stade localement avancé (IB2 à IVA) ayant eu une lymphadénectomie lombo-aortique de stadification quand le critère de positivité était un métabolisme ganglionnaire supérieur au bruit de fond de l'aorte sous-rénale. En considérant les examens négatifs et douteux, la valeur prédictive négative par patiente et par ganglion était alors, respectivement, de $96 \%$ et de 99,1\%. La lymphadénectomie lombo-aortique n'étant pas réalisée dans les cas où la TEP était positive, nous ne pouvions étudier la sensibilité, la spécificité et la valeur prédictive positive de celle-ci.

La valeur prédictive négative ganglionnaire lombo-aortique de la TEP/TDM varie de 94 à $83 \%$ dans la littérature avec toutefois des performances diminuées pour les métastases ganglionnaires de petite taille $\leq 5 \mathrm{~mm}$ (Tableau 4) $[10,11,33-$ 37]. Selon les études, l'incidence des micrométastases est évaluée entre 2 à $8 \%$ [38,39].

C'est pour cela qu'il existerait un risque d'évolutivité carcinologique en l'absence de traitement des patientes négatives en lombo-aortique en TEP/TDM, mais porteuses d'une atteinte métastatique ganglionnaire. Toutefois, si les champs de radiothérapie étaient étendus de façon systématique au rétropéritoine, de nombreuses patientes seraient alors surtraitées. En raison d'un taux non négligeable de métastases ganglionnaires $<5 \mathrm{~mm}$, on ne peut sursoir à la lymphadénectomie de stadification lombo-aortique en cas de TEP négative.

Ainsi, dans notre équipe et dans de nombreuses équipes françaises, un curage ganglionnaire lombo-aortique de stadification est proposé aux patientes atteintes d'un cancer

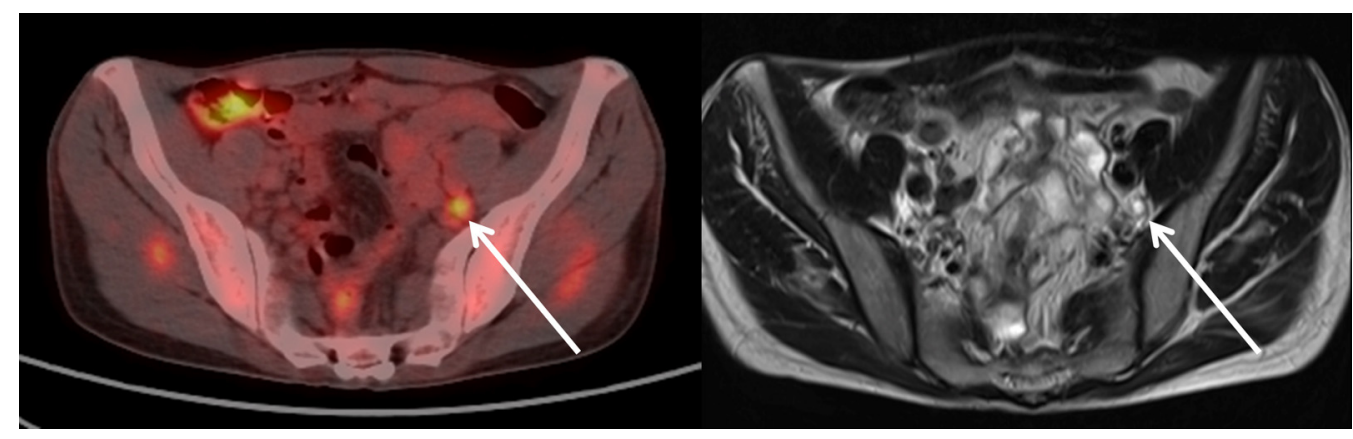

Fig. 6. Hypermétabolisme dans la région iliaque externe gauche en rapport avec une fixation physiologique ovarienne de ${ }^{18}$ FDG.

Hypermetabolism in the left external iliac area due to physiological ovarian uptake of ${ }^{18} \mathrm{FDG}$. 
Tableau 4

Étude de la TEP/TDM pour la détection des ganglions lombo-aortiques dans les cancers du col utérin localement avancés.

Studies of PET for detection of para-aortic lymph node metastasis in locally advanced cervical cancer.

\begin{tabular}{|c|c|c|c|c|c|c|c|c|c|c|c|c|c|c|}
\hline \multirow[t]{2}{*}{ Auteurs } & \multirow[t]{2}{*}{$n$} & \multirow[t]{2}{*}{ FIGO } & \multicolumn{2}{|l|}{ Critères } & \multirow{2}{*}{$\begin{array}{l}\text { Délai (moyen) } \\
\text { avant curage (j) }\end{array}$} & \multicolumn{4}{|l|}{ Nombre } & \multirow{2}{*}{$\begin{array}{l}\text { VPN } \\
(\%)\end{array}$} & \multirow{2}{*}{$\begin{array}{l}\text { VPP } \\
(\%)\end{array}$} & \multirow{2}{*}{$\begin{array}{l}\text { Sensibilité } \\
(\%)\end{array}$} & \multirow{2}{*}{$\begin{array}{l}\text { Spécificité } \\
(\%)\end{array}$} & \multirow{2}{*}{$\begin{array}{l}\text { Causes } \\
\text { de FN }\end{array}$} \\
\hline & & & Inclusion & $\begin{array}{l}\text { Ganglions } \\
\text { envahis }\end{array}$ & & TEP - & TEP + & $\mathrm{FN}$ & VP & & & & & \\
\hline Uzan (2011) [33] & 95 & IB2-IVA & $\begin{array}{l}\text { TEP négatif } \\
\text { en LA }\end{array}$ & AP & 16 & 95 & - & 8 & - & 82 & - & - & - & $5 \mathrm{ggl}<5 \mathrm{~mm}$ \\
\hline $\begin{array}{l}\text { Ramirez } \\
\text { (2011) [34] }\end{array}$ & 60 & IB2-IIIB & $\begin{array}{l}\text { TDM/IRM } \\
\text { négatif en LA }\end{array}$ & - & 3 & 53 & 7 & 9 & 5 & 83 & 71 & 36 & 96 & - \\
\hline $\begin{array}{l}\text { Boughanim } \\
\quad(2008) \text { [35] }\end{array}$ & 38 & IB2-II & $\begin{array}{l}\text { TEP négatif } \\
\text { en LA }\end{array}$ & AP & 123 & 38 & - & 3 & - & 92 & - & - & - & $2 \mathrm{ggl}<5 \mathrm{~mm}$ \\
\hline $\begin{array}{l}\text { Yildirim } \\
\qquad(2008)[36]\end{array}$ & 16 & IIB-IIIB & $\begin{array}{l}\text { TDM négatif } \\
\text { en LA }\end{array}$ & - & 8,3 & 12 & 4 & 2 & 2 & 83 & 50 & 50 & 83 & $2 \mathrm{MM}$ \\
\hline Loft (2007) [11] & 119 & IB1-IVA & $\begin{array}{l}\text { Tous les } \\
\text { patients }\end{array}$ & $\begin{array}{l}\text { AP/suivi/ } \\
\text { imagerie }\end{array}$ & - & 103 & 16 & 103 & 15 & 100 & 93 & 100 & 99 & - \\
\hline Lin (2002) [37] & 50 & IB2-IVA & $\begin{array}{l}\text { TDM négatif } \\
\text { en LA }\end{array}$ & AP & - & 36 & 14 & 2 & 12 & 94 & 86 & 86 & 94 & - \\
\hline Rose (1999) [10] & 32 & IIB-IVA & $\begin{array}{l}\text { TDM négatif } \\
\text { en LA }\end{array}$ & AP & 3 & 24 & 8 & 2 & 6 & 92 & 75 & 75 & 92 & $1 \mathrm{MM}$ \\
\hline
\end{tabular}

LA : lombo-aortique ; AP : anatomopathologie ; ggl : ganglion ; FN : faux négatifs ; VPN : valeur prédictive négative ; VP : vrais positifs ; VPP : valeur prédictive positive ; MM : micrométastases.

localement avancé dont la TEP/TDM est normale. Ce risque de micrométastases non diagnostiquées par TEP/TDM est à mettre en balance avec la morbi-mortalité du curage ganglionnaire. Il n'existe pas à ce jour d'étude comparant la place de la TEP dans l'évaluation ganglionnaire lombo-aortique par rapport à la stadification chirurgicale en termes de survie et d'effets secondaires.
Un des cas qui attire notre attention est l'une des 6 patientes n'ayant pas une de stadification chirurgicale lombo-aortique initiale en raison de comorbidités (Fig. 7). La TEP/TDM initiale retrouvait des ganglions positifs pelviens, mais pas de fixation pathologique ganglionnaire rétropéritonéale. Elle présentait un adénocarcinome du col utérin de stade IIB de FIGO traité par radiochimiothérapie concomitante et

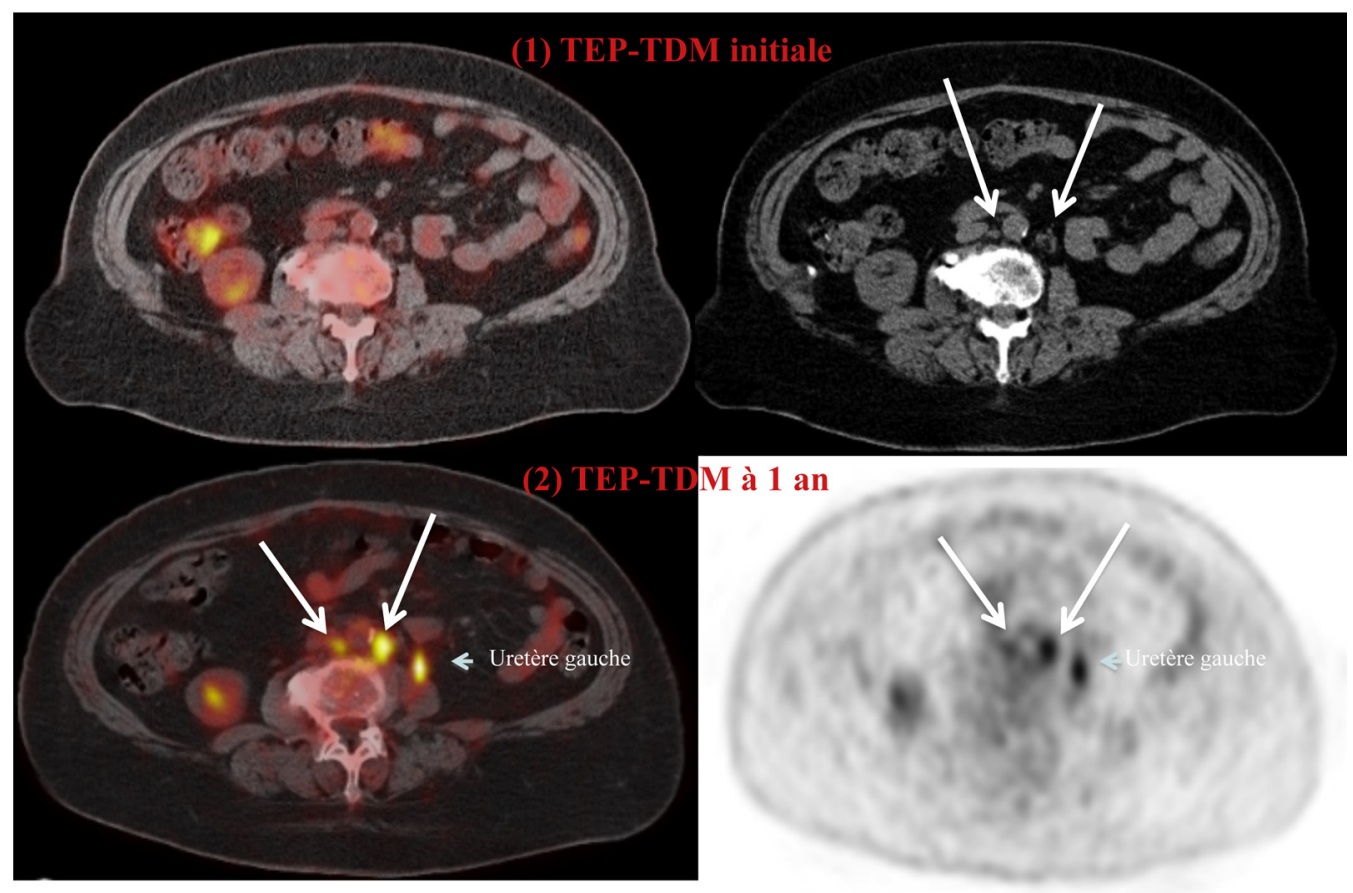

Fig. 7. Progression ganglionnaire lombo-aortique. La TEP/TDM initiale (1) retrouve 2 ganglions mesurant moins de $10 \mathrm{~mm}$ de petit axe sans fixation pathologique de ${ }^{18}$ FDG. La TEP/TDM de surveillance à 1 an (2) retrouve un hypermétabolisme intéressant les 2 ganglions précédemment décrits suspects de ganglions métastatiques : 1 ganglion inter-aortico-cave $\left(\mathrm{SUV}_{\max }=4,3\right.$ et $7 \mathrm{~mm}$ de petit axe) et 1 ganglion latéro-aortique (SUV $\max =7,9$ et $9 \mathrm{~mm}$ de petit axe).

Para-aortic lymph node progression. Initial PET/CT (1) shows 2 lymph nodes less than 10 mm in short-axis without ${ }^{18}$ FDG uptake. One year monitoring PET/CT(2) 
Tableau 5

Proposition d'indication du curage lombo-aortique en fonction des résultats de la TEP/TDM

Indication proposal of para-aortic lymphadenectomy according to PET/CT results.

\begin{tabular}{lll}
\hline TEP en pelvien & $\begin{array}{l}\text { TEP en } \\
\text { lombo-aortique }\end{array}$ & $\begin{array}{l}\text { Indication de curage } \\
\text { lombo-aortique }\end{array}$ \\
\hline Négatif & Négatif & $\begin{array}{l}\text { Oui, mais tenir compte } \\
\text { de la morbidité et des antécédents }\end{array}$ \\
Positif & Négatif & $\begin{array}{l}\text { Oui } \\
\text { Non, car peu de faux positifs } \\
\text { Positif }\end{array}$ \\
Négatif & Positif & Oui, situation très rare \\
\hline
\end{tabular}

curiethérapie puis chirurgie de clôture. Le délai entre la TEP/ TDM et le début de la radiothérapie a été de 28 jours. Un an après, cette patiente a présenté une récidive ganglionnaire lombo-aortique. La TEP a pu méconnaître des micrométastases qui, en l'absence de traitement local par radiothérapie, ont progressé. Les deux arguments en faveur de la présence de micrométastases au stade initial sont que les deux ganglions étaient présents un an auparavant mais non hypermétaboliques et morphologiquement rassurant et, deuxièmement, que la patiente était bonne répondeuse à l'étage pelvien après traitement.

Lorsqu'il existe des ganglions positifs en TEP/TDM en région lombo-aortique et également en région pelvienne, une extension du champ de radiothérapie en lombo-aortique est indiquée sans vérification histologique en raison de la très bonne valeur prédictive positive de la TEP/TDM.

Dans les rares cas de fixation lombo-aortique sans atteinte pelvienne, une lymphadénectomie est indiquée afin d'éliminer les faux positifs. Dans notre série, les 9 patientes qui présentaient une atteinte ganglionnaire lombo-aortique avaient une atteinte pelvienne associée (Tableau 5).

\section{Conclusion}

Notre étude démontre la valeur pronostique de la TEP-FDG. La SUV ${ }_{\max }$ de la tumeur cervicale apparaît différente entre les stades FIGO I versus II et IV, ainsi que chez les patientes en réponse tumorale complète comparativement à celles en réponse partielle après radiochimiothérapie concomitante. Néanmoins, des études complémentaires seront nécessaires pour valider ces résultats à propos d'effectifs plus conséquents.

La $\mathrm{SUV}_{\max }$ de la tumeur primitive du col est corrélée au grand axe de la tumeur et représenterait de façon indirecte un marqueur de la prolifération cellulaire et de la différenciation tumorale.

Cette étude confirme la très bonne valeur prédictive négative de la TEP dans l'évaluation ganglionnaire lombo-aortique des cancers localement avancés. À l'heure actuelle, dans de nombreuses équipes, la lymphadénectomie lombo-aortique est réalisée chez les patientes dont la TEP est négative afin d'éliminer toute atteinte métastatique de petite taille que la TEP aurait pu ignorer. Il serait intéressant d'étudier la place de la TEP dans l'évaluation ganglionnaire par rapport à la stadification chirurgicale en termes de survie et d'effets secondaires.

Pour les cancers de stades localisés, le risque de micrométastases ganglionnaires n'est pas négligeable et ne permet pas de surseoir à une lymphadénectomie pelvienne de stadification. La technique du ganglion sentinelle pourrait représenter une alternative au curage systématique. La stadification pourrait être améliorée alors grâce aux techniques histologiques d'immunohistochimie et de coupes sériées sur les quelques ganglions prélevés.

\section{Déclaration d'intérêts}

Les auteurs déclarent ne pas avoir de conflits d'intérêts en relation avec cet article.

\section{Références}

[1] Pecorelli S, Zigliani L, Odicino F. Revised FIGO staging for carcinoma of the cervix. Int J Gynaecol Obstet 2009;105:107-8.

[2] Choi HJ, Ju W, Myung SK, Kim Y. Diagnostic performance of computer tomography, magnetic resonance imaging, and positron emission tomography or positron emission tomography/computer tomography for detection of metastatic lymph nodes in patients with cervical cancer: metaanalysis. Cancer Sci 2010;101:1471-9.

[3] Resbeut M, Fondrinier E, Fervers B, Haie-Meder C, Bataillard A, Lhommé C, et al. Standards, options and recommendations for the management of invasive cervical cancer patients (non metastastic). Bull Cancer 2003;90:333-46.

[4] Park W, Park YJ, Huh SJ, Kim BG, Bae DS, Lee J, et al. The usefulness of MRI and PET imaging for the detection of parametrial involvement and lymph node metastasis in patients with cervical cancer. Jpn J Clin Oncol 2005;35:260-4.

[5] Lee Y-Y, Choi CH, Kim CJ, Kang H, Kim T-J, Lee J-W, et al. The prognostic significance of the SUVmax (maximum standardized uptake value for F-18 fluorodeoxyglucose) of the cervical tumor in PET imaging for early cervical cancer: preliminary results. Gynecol Oncol 2009; 115:65-8.

[6] Leseur J, Devillers A, Williaume D, Le Prisé E, Fougerou C, Bouriel C, et al. 18F-fluorodeoxyglucose PET/CT in cervix cancer: lymph node assessment and prognostic/predictive value of primary tumour analysis. Cancer Radiother 2011;15:699-708.

[7] Kidd EA, Siegel BA, Dehdashti F, Grigsby PW. The standardized uptake value for F-18 fluorodeoxyglucose is a sensitive predictive biomarker for cervical cancer treatment response and survival. Cancer 2007;110:173844.

[8] Xue F, Lin LL, Dehdashti F, Miller TR, Siegel BA, Grigsby PW. F18 fluorodeoxyglucose uptake in primary cervical cancer as an indicator of prognosis after radiation therapy. Gynecol Oncol 2006;101:147-51.

[9] Société française d'oncologie gynécologique (SFOG). Prise en charge initiale des cancers gynécologiques : référentiels de la Société française d'oncologie gynécologique [online]; 2010, Disponible sur : http://asfog.free.fr/images/stories/PDF/referentiel_sfog.pdf.

[10] Rose PG, Adler LP, Rodriguez M, Faulhaber PF, Abdul-Karim FW, Miraldi F. Positron emission tomography for evaluating para-aortic nodal metastasis in locally advanced cervical cancer before surgical staging: a surgicopathologic study. J Clin Oncol 1999;17:41-5.

[11] Loft A, Berthelsen AK, Roed H, Ottosen C, Lundvall L, Knudsen J, et al. The diagnostic value of PET/CT scanning in patients with cervical cancer: a prospective study. Gynecol Oncol 2007;106:29-34.

[12] Yen T-C, See L-C, Lai C-H, Yah-Huei CW, Ng K-K, Ma S-Y, et al. 18FFDG uptake in squamous cell carcinoma of the cervix is correlated with glucose transporter 1 expression. J Nucl Med 2004;45:22-9. 
[13] Park SI, Suh DS, Kim S-J, Choi KU, Yoon M-S. Correlation between biological marker expression and F-fluorodeoxyglucose uptake in cervical cancer measured by positron emission tomography. Onkologie 2013;36:169-74.

[14] Merlin C, Cachin F, Kelly A, Mestas D, De Freitas D, Maublant J. Prediction of lymph node status in uterine cervical cancer with 18FDG-PET/CT-value of primary tumor uptake. Med Nucl 2008;32:313-66.

[15] Pan L, Cheng J, Zhou M, Yao Z, Zhang Y. The SUVmax (maximum standardized uptake value for F-18 fluorodeoxyglucose) and serum squamous cell carcinoma antigen ( $\mathrm{SCC}-\mathrm{ag}$ ) function as prognostic biomarkers in patients with primary cervical cancer. J Cancer Res Clin Oncol 2012;138:239-46.

[16] Miller TR, Grigsby PW. Measurement of tumor volume by PET to evaluate prognosis in patients with advanced cervical cancer treated by radiation therapy. Int J Radiat Oncol Biol Phys 2002;53:353-9.

[17] Kidd EA, Spencer CR, Huettner PC, Siegel BA, Dehdashti F, Rader JS, et al. Cervical cancer histology and tumor differentiation affect $18 \mathrm{~F}$ fluorodeoxyglucose uptake. Cancer 2009;115:3548-54.

[18] Nguyen XC, Lee WW, Chung J-H, Park SY, Sung SW, Kim YK, et al. FDG uptake, glucose transporter type 1, and Ki-67 expressions in non-smallcell lung cancer: correlations and prognostic values. Eur J Radiol 2007;62:214-9

[19] Kawana H, Tamaru J, Tanaka T, Hirai A, Saito Y, Kitagawa M, et al. Role of p27Kip1 and cyclin-dependent kinase 2 in the proliferation of nonsmall cell lung cancer. Am J Pathol 1998;153:505-13.

[20] Buck AC, Schirrmeister HH, Guhlmann CA, Diederichs CG, Shen C, Buchmann I, et al. Ki-67 immunostaining in pancreatic cancer and chronic active pancreatitis: does in vivo FDG uptake correlate with proliferative activity? J Nucl Med 2001;42:721-5.

[21] Morice P, Castaigne D, Pautier P, Rey A, Haie-Meder C, Leblanc M, et al. Interest of pelvic and paraaortic lymphadenectomy in patients with stage IB and II cervical carcinoma. Gynecol Oncol 1999;73:106-10.

[22] Kidd EA, Siegel BA, Dehdashti F, Rader JS, Mutch DG, Powell MA, et al. Lymph node staging by positron emission tomography in cervical cancer: relationship to prognosis. J Clin Oncol 2010;28:2108-13

[23] Kim SH, Kim SC, Choi BI, Han MC. Uterine cervical carcinoma: evaluation of pelvic lymph node metastasis with MR imaging. Radiology 1994;190:807-11.

[24] Houdebine S, Ricaud-Couprie M, Labbe-Devilliers C, Doutriaux-Dumoulin I, Geffroy D, Meingan P. Value of MRI for pelvic imaging: normal and pathological aspects. Med Nucl 2012;36:451-8.

[25] Morisawa N, Koyama T, Togashi K. Metastatic lymph nodes in urogenital cancers: contribution of imaging findings. Abdom Imaging 2006;31:620-9.

[26] Juretzka MM, Jensen KC, Longacre TA, Teng NN, Husain A. Detection of pelvic lymph node micrometastasis in stage IA2-IB2 cervical cancer by immunohistochemical analysis. Gynecol Oncol 2004;93:107-11.

[27] Fregnani JHTG, Latorre MRDO, Novik PR, Lopes A, Soares FA. Assessment of pelvic lymph node micrometastatic disease in stages IB and IIA of carcinoma of the uterine cervix. Int J Gynecol Cancer 2006;16:1188-94.
[28] Horn L-C, Hentschel B, Fischer U, Peter D, Bilek K. Detection of micrometastases in pelvic lymph nodes in patients with carcinoma of the cervix uteri using step sectioning: frequency, topographic distribution and prognostic impact. Gynecol Oncol 2008;111:276-81.

[29] Sironi S, Buda A, Picchio M, Perego P, Moreni R, Pellegrino A, et al. Lymph node metastasis in patients with clinical early-stage cervical cancer: detection with integrated FDG PET/CT. Radiology 2006;238:272-9.

[30] Chou H-H, Chang T-C, Yen T-C, Ng K-K, Hsueh S, Ma S-Y, et al. Low value of [18F]-fluoro-2-deoxy-D-glucose positron emission tomography in primary staging of early-stage cervical cancer before radical hysterectomy. J Clin Oncol 2006;24:123-8.

[31] Lécuru F, Mathevet P, Querleu D, Leblanc E, Morice P, Daraï E, et al. Bilateral negative sentinel nodes accurately predict absence of lymph node metastasis in early cervical cancer: results of the SENTICOL study. J Clin Oncol 2011:29:1686-91.

[32] Lerman H, Metser U, Grisaru D, Fishman A, Lievshitz G, Even-Sapir E. Normal and abnormal 18F-FDG endometrial and ovarian uptake in preand postmenopausal patients: assessment by PET/CT. J Nucl Med 2004;45:266-71.

[33] Uzan C, Souadka A, Gouy S, Debaere T, Duclos J, Lumbroso J, et al. Analysis of morbidity and clinical implications of laparoscopic para-aortic lymphadenectomy in a continuous series of 98 patients with advancedstage cervical cancer and negative PET-CT imaging in the para-aortic area. Oncologist 2011;16:1021-7.

[34] Ramirez PT, Milam MR. Laparoscopic extraperitoneal paraaortic lymphadenectomy in patients with locally advanced cervical cancer. Gynecol Oncol 2007;104:9-12.

[35] Boughanim M, Leboulleux S, Rey A, Pham CT, Zafrani Y, Duvillard P, et al. Histologic results of para-aortic lymphadenectomy in patients treated for stage IB2/II cervical cancer with negative [18F]fluorodeoxyglucose positron emission tomography scans in the para-aortic area. J Clin Oncol 2008;26:2558-61.

[36] Yildirim Y, Sehirali S, Avci ME, Yilmaz C, Ertopcu K, Tinar S, et al. Integrated PET/CT for the evaluation of para-aortic nodal metastasis in locally advanced cervical cancer patients with negative conventional CT findings. Gynecol Oncol 2008;108:154-9.

[37] Lin WC, Hung YC, Yeh LS, Kao CH, Yen RF, Shen YY. Usefulness of (18)F-fluorodeoxyglucose positron emission tomography to detect para-aortic lymph nodal metastasis in advanced cervical cancer with negative computed tomography findings. Gynecol Oncol 2003;89: $73-6$.

[38] Zand B, Euscher ED, Soliman PT, Schmeler KM, Coleman RL, Frumovitz M, et al. Rate of para-aortic lymph node micrometastasis in patients with locally advanced cervical cancer. Gynecol Oncol 2010;119:422-5.

[39] Martínez A, Mery E, Ferron G, Querleu D. Incidence of micrometastases in histologically negative para-aortic lymph nodes in advanced cervica cancer patients. Gynecol Oncol 2010;119:76-80. 